\title{
Satellitengestützte Lokalisierung in urbanen Gebieten mit globalen Satellitennavigationssystemen
}

\author{
P. Reisdorf und G. Wanielik \\ Professur für Nachrichtentechnik, Technische Universität Chemnitz, Reichenhainer Straße 70, 09126 Chemnitz, Germany \\ Correspondence to: P. Reisdorf (pierre.reisdorf@etit.tu-chemnitz.de)
}

Zusammenfassung. Die Kenntnis des eigenen Standortes an jedem Punkt der Erde und zu jeder Zeit ist eine wichtige Voraussetzung für viele Anwendungen sowohl im Straßenverkehr als auch zur Orientierung einzelner Personen. Wurde bisher das bekannte GPS vorwiegend eingesetzt, so existiert mit GLONASS und entsprechenden Mehrsystemempfängern die Möglichkeit der Nutzung von mehreren Satellitensystemen. Dadurch erhöht sich augenblicklich die Anzahl zur Verfügung stehender Satelliten, wodurch sich bestimmte Positionierungseigenschaften wie Verfügbarkeit, Integrität und Genauigkeit verbessern lassen.

\section{Einführung}

Die Positionierung mit Satellitensystemen bildet heute eine einfache Möglichkeit der Lokalisierung an jedem Punkt der Erde mit einem entsprechenden Empfänger. Dabei haben sich bedingt durch unterschiedliche Interessen mehrere Satellitensysteme entwickelt. Neben dem bekannten Global Positioning System (GPS) existieren noch weitere globale Systeme, wie GLONASS oder Galileo.

Jedes System bietet bei optimalen Empfangsbedingungen gute Positionslösungen, die für viele Einsatzzwecke auch ausreichend sind. Innerhalb eines urbanen Gebietes ergeben sich jedoch erhebliche Einschränkungen in den Empfangsbedingungen, weshalb die zu erwartenden Positionslösungen unbefriedigend sind. Dies hat zum einen die Ursache in der Abschattung von Satelliten, die durch andere Objekte verdeckt sind und dadurch nicht mehr empfangen werden können. Zum anderen bekommen noch weitere Fehler, wie Mehrwegeeffekte, einen größeren Einfluss. Ein urbanes Gebiet zeichnet sich dabei durch vom Menschen künstlich geschaffene Objekte aus, die eine Sichtbarriere zwischen einem Satelliten und einem Empfänger bilden können.

Durch die Existenz unterschiedlicher Satellitensysteme, die vorwiegend einzeln für sich genutzt werden, lässt sich

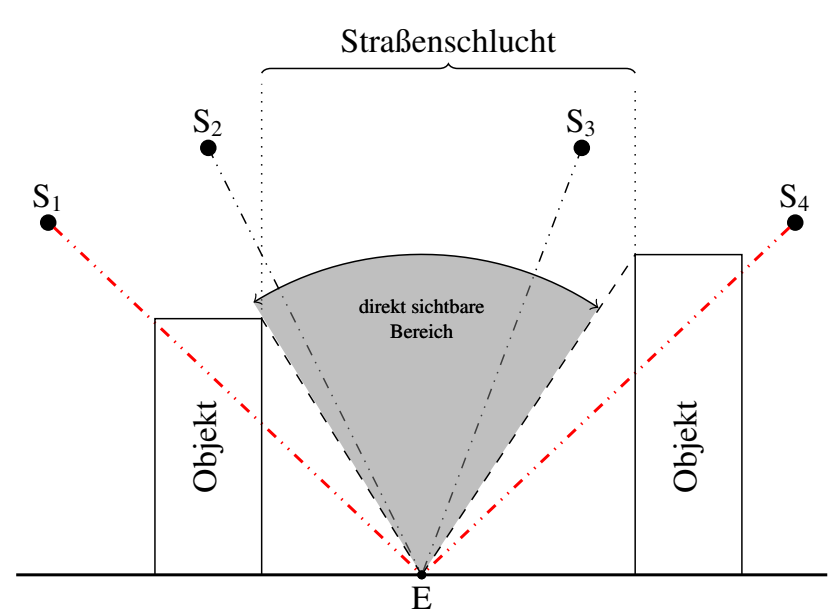

Abb. 1. Szene in einem urbanen Gebiet mit einer Straßenschlucht. Die Markierungen $\mathrm{S}_{1 . .4}$ stehen für Standorte von unterschiedlichen Satelliten zu einem Zeitpunkt. Die Sichtverbindung zu den Satelliten sind zum einen möglich $\left(\mathrm{S}_{2}\right.$ und $\left.\mathrm{S}_{3}\right)$ und zum anderen durch Objekte verdeckt $\left(\mathrm{S}_{1}\right.$ und $\left.\mathrm{S}_{4}\right)$.

durch das Zusammenführen der Systeme als ein Gesamtsystem dem Problem der Abschattung einzelner Satelliten begegnen. Dadurch erhöht sich die Anzahl der zur Verfügung stehenden Satelliten, wodurch nur algorithmisch Anpassungen getätigt werden müssen. In dieser Arbeit werden die beiden Satellitensysteme GPS und GLONASS zur Positionsberechnung als ein Satellitensystem betrachtet, womit sich die Positionslösungen im urbanen Gebiet gegenüber den Einzelsystemen verbessern. Abbildung 1 verdeutlicht schematisch die Szenerie mit der beschränkten Sichtbarkeit der Satelliten im urbanen Gebiet.

Im zweiten Kapitel werden ähnliche Arbeiten und der Stand der Technik beschrieben. Darauf folgend werden kurz markante Unterschiede zwischen den Satellitensystemen 
GPS und GLONASS aufgezeigt. Im vierten Kapitel wird auf die Grundlagen zur Positionsbestimmung mit Satelliten eingegangen. Im fünften Kapitel wird auf die Evaluationsmethodik und die zu untersuchenden Parameter eingegangen. Abschließend werden die quantitativen Ergebnisse ausgewertet und zum Schluss zusammengefasst.

\section{Stand der Technik \& Ähnliche Arbeiten}

Mit der Entstehung unterschiedlicher Satellitensysteme und der stetigen Entwicklung der einzelnen Systeme kam die Idee, die Systeme miteinander zu verknüpfen. Durch die mehr oder weniger verfügbare Anzahl an verwendbaren Satellitensignalen wurde bei vielen Arbeiten überwiegend auf Daten aus Simulatoren zurückgegriffen. Einer dieser Simulatoren ist der Galileo System Simulation Facility (GSSF), ein anderer der Navys Artaud et al. (2010). Mit diesen Programmen lassen sich unterschiedliche Szenarien anhand von echten Bahndaten - werden im Internet zur Verfügung gestellt - erzeugen, Daten visualisieren und auswerten. Das Ziel der Verbindung von unterschiedlichen Satellitensystemen ist zum einen die verschiedenen Fehlereinflüsse zu minimieren und zum anderen Eigenschaften wie Verfügbarkeit, Genauigkeit und Integrität zu verbessern.

Für die Fehlereinflüsse gibt es unterschiedliche Konzepte wie die Verringerung des Zeitoffsets zwischen zwei Systemen Juang and Tsai (2009); Xu et al. (2011); Cai (2008). Das Offset zwischen den Zeitsystemen muss bei Unkenntnis zusätzlich geschätzt werden und bedingt dadurch einen zusätzlichen Satelliten für die Korrektur des Offsets. Weitere Möglichkeiten zur Minimierung von Fehlern sind die Nutzung von Filtern wie das Kalman Filter Delmas et al. (2008) oder auch einen Partikel-Filter Nahimana et al. (2008).

In den unterschiedlichen Arbeiten werden teilweise zwei Systeme betrachtet, wie GPS und Galileo Engel (2008) oder GPS und GLONASS Mattos (2011). Auf mehr als zwei Systeme wird in Rizos (2008) eingegangen, jedoch setzen die Ergebnisse auf Simulationsdaten auf.

Ein Grund zur Erweiterung von einem Satellitensystem um andere Systeme sind eingeschränkte Empfangsbedingungen und Störungen der Signale wie in einem urbanen Gebiet. Die Verfügbarkeit und die Genauigkeit wurden teilweise bei Mattos (2011) für GPS und GLONASS sowie für GPS und QZSS betrachtet. Ein anderer Ansatz der Verbesserung in urbanen Gebieten ist die Verwendung von zusätzlichen Sensoren wie Inertialsensoren Duchateau et al. (2009).

Die Positionslösungen sind abhängig von der Konstellation der Satelliten zum Empfänger. Durch die erhöhte Anzahl der Satelliten bei der Zusammenlegung von mehreren Systemen besteht die Möglichkeit der selektiven Auswahl von Satelliten für eine geeignete Konstellation. In Miaoyan et al. (2008) wird ein Verfahren zur Auswahl von Satelliten mit einer optimalen Konstellation vorgestellt.
Tabelle 1. Eigenschaften von GPS und GLONASS

\begin{tabular}{lcc}
\hline Eigenschaft & GPS & GLONASS \\
\hline $\begin{array}{l}\text { Koordinatensystem } \\
\text { Zeitsystem }\end{array}$ & WGS84 & PZ-90.02 \\
Navigationsnachricht & eigene Atomzeit \\
Bahndaten & unterschiedliche Beschreibung \\
& Kepler-Parameter & $\begin{array}{c}\text { geozentrische Koordinaten } \\
\text { mit ihren Abteilungen }\end{array}$ \\
\hline
\end{tabular}

\section{Satellitensysteme}

Aktuell existieren zwei voll ausgebaute, globale Satellitennavigationsysteme (GNSS). Das sind zum einen das amerikanische GPS und zum anderen das russiche GLONASS. Der Grundaufbau mit einem Raum-, Boden-, und Nutzersegment sowie das Prinzip der Ortung sind bei beiden Systemen ähnlich. Im Detail existieren jedoch Unterschiede, die in Tabelle 1 aufgeführt sind. Beide Systeme besitzen unterschiedliche Koordinaten- und Zeitsysteme, welche sich mit leichten Unsicherheiten ineinander überführen lassen. Die Navigationsnachrichten haben eine unterschiedliche Struktur und Informationsdichte. Algorithmisch am ausgeprägtesten ist der Unterschied in der Bahnbeschreibung beider Satellitensysteme. GPS verwendet Kepler-Parameter, wohingegen GLONASS geozentrische Koordinaten mit ihren Ableitungen einsetzt. Für die Verwendung beider Systeme zu einem Gesamtsystem muss bei den wählbaren Eigenschaften wie Koordinaten- und Zeitsystem ein bevorzugtes System gewählt werden. Da GPS weiter verbreitet und dadurch besser untersucht ist, wurden die wählbaren Parameter zugunsten von GPS gewählt.

\section{Grundlagen}

\subsection{GNSS Positionierung}

Die Funktionsweise der Positionierung mit Satellitensystemen beruht auf dem Time of arrival (TOA) Prinzip, also die Bestimmung der Entfernung durch Messung der Signallaufzeit von Funkwellen. Die Satelliten senden kontinuierlich Funkwellen mit einer aufmodulierten Zeit. Ein Empfänger kann anhand der aufmodulierten Zeit, der Empfangszeit und der Kenntnis des Ortes des Satelliten zum Zeitpunkt der Aussendung der Funkwellen die Entfernung bestimmen Kaplan and Hegarty (2006). Durch die unterschiedlichen Uhren im Empfänger und der Satellitensystemzeit ergibt sich ein Uhrzeitfehler, der abhängig vom Empfänger und innerhalb dessen in jeder Messung enthalten ist. Die Entfernung zwischen dem Empfänger und einem Satelliten wird folglich nicht als wahre Entfernung $r$, sondern als Pseudoentfernung $\rho$ bezeichnet. Gleichung (1) verdeutlicht den Zusammenhang. Die Pseudoentfernung ergibt sich aus der wahren Entfernung $r$ und dem Uhrzeitenfehler (zwischen Empfänger und der Systemzeit) mal der Lichtgeschwindigkeit $c$. Zusätzlich 
existieren noch weitere Fehler, bezeichnet als $d_{\mathrm{e}}$, die zu berücksichtigen sind. Diese sind tropos- oder ionosphärische Fehler sowie Ephemeridenfehler, Messfehler und weitere, auf die jedoch nicht weiter eingegangen wird. Da der Empfängeruhrzeitfehler in jeder Messung enthalten ist, kann dieser mit einer zusätzlichen Messung zu einem Satelliten geschätzt und beseitigt werden. Dadurch werden im Minimum vier Satelliten für eine Positionsbestimmung im Raum benötigt - siehe Gleichung (2).

$\rho=r+c(d t-d T)+d_{\mathrm{e}}$

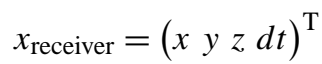

\subsection{Mehrsystem GNSS Positionierung}

Unter Verwendung von mehr als einem System ergibt sich zusätzlich noch ein weiterer Uhrzeitfehler. Dieser ist systembedingt und kann nur durch einen weiteren Satelliten geschätzt werden. Dadurch erweitert sich unser Ergebnisvektor um $d t_{\text {GLONASS }}$ und es werden im Minimum für eine Positionsbestimmung im Raum der Empfang von fünf zeitgleichen Messungen zu unterschiedlichen Satelliten nötig.

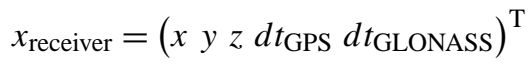

\section{Evaluationsmethodik}

Für die Entwicklung wurde das Framework der Professur Nachrichtentechnik der TU Chemnitz verwendet und erweitert. Dieses basiert auf der Sprache C\# und ermöglicht eine komponentenbasierte Entwicklung von Algorithmen sowie funktional die Möglichkeit der rapid prototyp Entwicklung von Advanced Driver Assistance Systems (ADASs).

Zur Evaluierung des entwickelten Systems wurden echte Messdaten aufgenommen, welche im Postprocessing mit den entwickelten Algorithmen und der Referenzmessung ausgewertet wurden. Zum Vergleich wurden jeweils die Einzelsysteme mit der Referenzmessung und die Einzelsysteme in $\mathrm{Zu}$ sammenführung als ein Gesamtsystem mit der Referenzmessung verglichen.

\subsection{Simulation Straßenschlucht}

In einem urbanen Gebiet ergeben sich durch bauliche Gegebenheiten sehr große Elevationswinkel. In dem evaluierten Gebiet ergaben sich nicht die gewünschten großen Elevationswinkel, da die Gebäude wesentlich kleiner sind. Dieser Umstand wurde softwareseitig mit der nachträglichen Vergrößerung des Elevationswinkels umgangen. Die Abbildung 2 zeigt den stetigen Anstieg des Winkels und der damit verbundenen Verkleinerung des sichtbaren Bereiches.

\subsection{Sensorkonfiguration}

Für die Überprüfung eines urbanen Mehrsystemszenarios wurden Echtdaten mit einem Messfahrzeug Carai Schubert

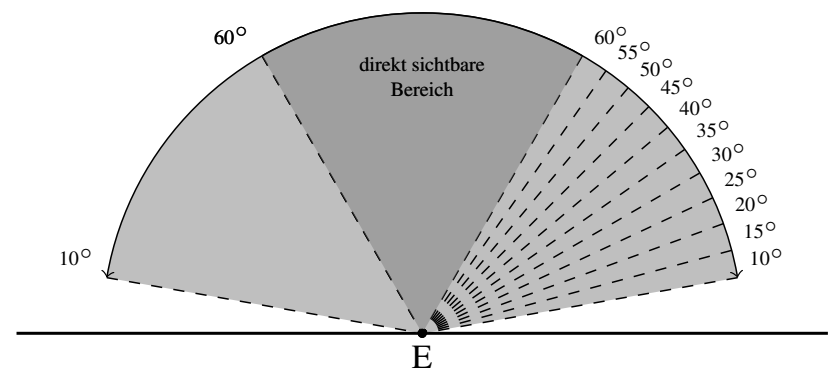

Abb. 2. Simulation einer Straßenschlucht durch sukzessive Vergrößerung des Elevationswinkels von $10^{\circ}-60^{\circ}$ und der damit verbundenen Verkleinerung des direkt sichtbaren Bereiches. Die Einschränkung geschieht parallel auf beiden Seiten des Empfängers vom Horizont aus gesehen.

et al. (2010) von der Professur Nachrichtentechnik der TU Chemnitz aufgenommen. Das Messfahrzeug beinhaltet einen NovAtel OEMV GNSS Empfänger mit der Unterstützung von GPS- und GLONASS-Rohdaten. Die Pseudoentfernungen wurden bei beiden Systemen mit $10 \mathrm{~Hz}$ aufgenommen. Für die Evaluation der erhaltenen Lösung wird eine hochgenaue Referenz (Ground truth) benötigt. Hierfür wird ein NovAtel SPAN System mit GNSS Positionierung verwendet. Dieses beinhaltet einen Mehrfrequenzempfänger für GPS mit Real Time Kinematic (RTK) Unterstützung. Dadurch wird eine Genauigkeit für die Referenzmessung im Zentimeterbereich erreicht.

\subsection{Evaluationskriterien}

Zum Vergleich möglicher Lösungen sind die Kriterien Verfügbarkeit, Genauigkeit und Integrität entscheidende Faktoren. Die Verfügbarkeit - Gleichung (4) und (5) - eines Systems ist größer, je mehr Satelliten zum Zeitpunkt der Messung zur Verfügung stehen.

$\bar{x}=\frac{1}{n} \sum_{i=1}^{n} x_{i}$

Mögliche Fixe $=\frac{n_{\text {Fixe }}}{n_{\text {Messungen }}}, \quad n \ldots$ Anzahl

Für die Genauigkeit wird der Root Mean Square Error (RMSE) - Gleichung (6) - als Vergleichsmaß verwendet. Bei diesem Maß werden Ausreißer im Ergebnis stärker gewichtet, was für die Erkennung von großen Fehlern von Vorteil ist.

$\mathrm{RMSE}=\sqrt{\frac{1}{n} \sum_{i=1}^{n}\left(f_{i}\right)^{2}}$

Die Integrität ergibt sich aus der Kovarianzmatrix der berechneten Lösung. Dabei gibt es verschiedene Konfidenzintervalle $(1 \sigma-3 \sigma)$, die in Abhängigkeit von der möglichen 


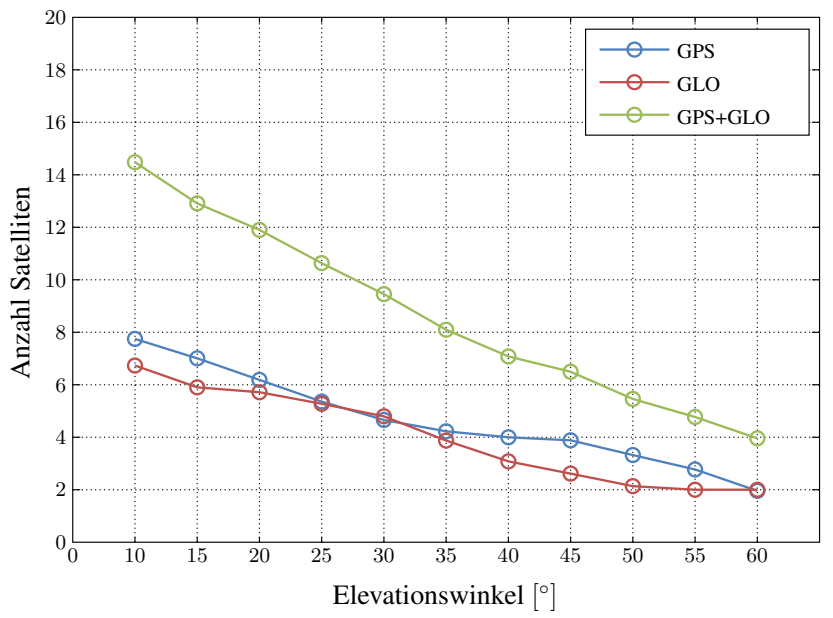

Abb. 3. Mittlere Anzahl verfügbarer GPS (blau), GLONASS (rot), GPS und GLONASS (grün) Satelliten in Abhängigkeit des Elevationswinkels.

Verwendung des Systems einzuhalten sind.

$$
\sigma=\sqrt{\operatorname{Cov}\left(X_{x}, X_{x}\right)+\operatorname{Cov}\left(X_{y}, X_{y}\right)+\operatorname{Cov}\left(X_{z}, X_{z}\right)}
$$

$\operatorname{Cov}() \ldots$ Kovarianz

\section{Quantitative Ergebnisse}

\subsection{Verfügbarkeit}

Die Erhöhung der Satellitenanzahl durch Zusammenführung beider Systeme zu einem System ist leicht nachvollziehbar (Abbildung 3). Die Anzahl erhöht sich ohne zusätzliche Änderungen an einem System. Die Folge der Vergrößerung der verfügbaren Satelliten wird in Abbildung 4 ersichtlich. Mit steigendem Elevationswinkel wird die Anzahl möglicher Fixe (Positionslösungen) bei den Einzelystemen stetig kleiner (blau und rot). Werden beide Systeme als ein System verwendet ergibt sich bei größeren Elevationswinkeln länger die Möglichkeit einer Positionsberechnung. Dadurch steht das System wesentlich länger zur Verfügung. Ein positiver Nebeneffekt ist zusätzlich, dass durch die größere Anzahl an Satelliten mehr Satelliten ausfallen oder bei der Positionsbestimmung ausgeschlossen werden können (Beispiel: Mehrwegeempfang).

\subsection{Positionsgenauigkeit}

Ein weiteres Kriterium neben der Verfügbarkeit ist die Genauigkeit der Positionsangabe. In Abbildung 5 ist dafür der RMSE jeweils über eine gesamte Messsequenz für einen bestimmten Elevationswinkel berechnet wurden. $\mathrm{Zu}$ erkennen ist in dieser Sequenz, dass GPS bei kleinen Elevationswinkeln eine bessere Genauigkeit bietet als GLONASS oder beide Systeme zusammen. Ab einem Elevationswinkel von $35^{\circ}$

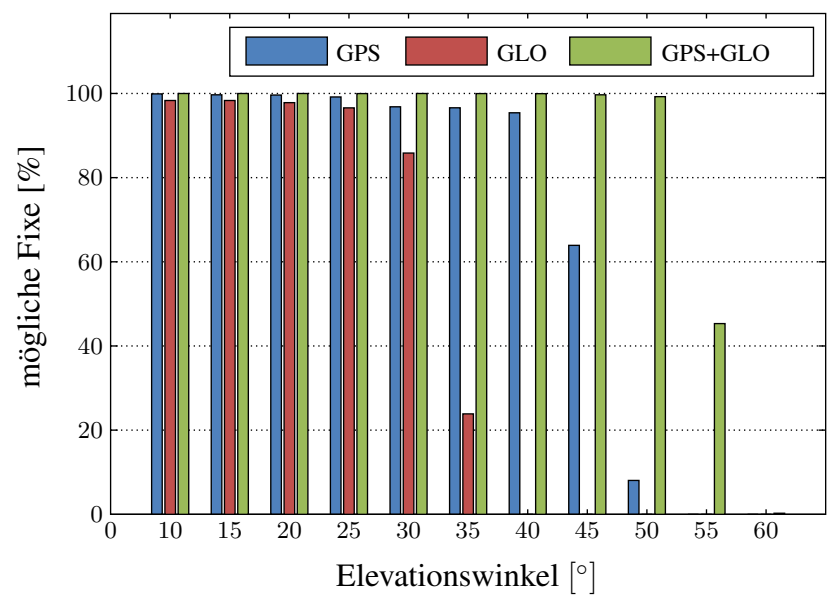

Abb. 4. Prozentuale Anzahl der möglichen Fixe für GPS (blau), GLONASS (rot), GPS und GLONASS (grün) ausgehend von der Anzahl möglicher Fixe der Referenzmessung.

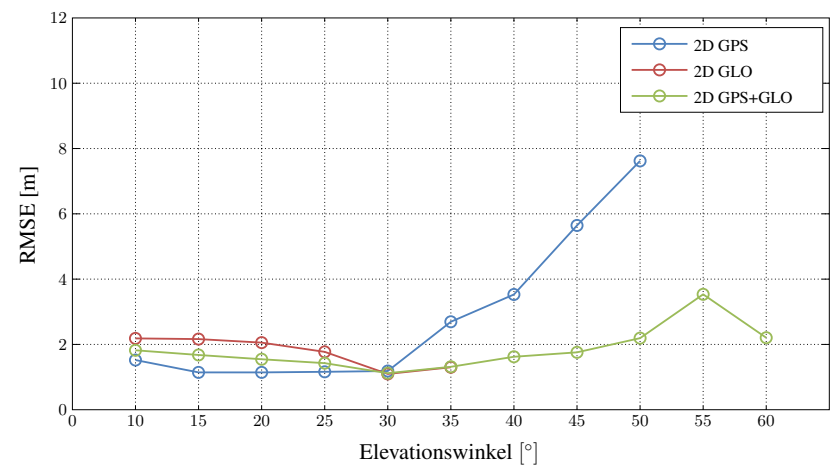

Abb. 5. Der RMSE für eine 2-D-Positionslösung in Abhängigkeit eines steigenden Elevationswinkels. Für GPS (blau), GLONASS (rot) sowie GPS und GLONASS zusammen wird eine Verbesserung hauptsächlich bei großen Elevationswinkeln deutlich.

steigt der RMSE für GPS jedoch deutlich an, wohingegen dieser für beide Systeme zusammen wesentlich kleiner bleibt und auch langsamer ansteigt.

Für GLONASS gab es in dieser Sequenz ab einem Elevationswinkel von $35^{\circ}$ keine Positionslösungen mehr. Bei der Verwendung beider Systeme als Gesamtsystem konnten jedoch einzelne GLONASS-Satelliten noch verwendet werden.

\subsection{Integrität}

Neben den ersten beiden Kriterien ist die Integrität noch von entscheidender Bedeutung. Mittels dieser lässt sich eine Aussage treffen, inwieweit dem Ergebnis vertraut werden kann. Abbildung 6 zeigt den 2-D-Positionsfehler und das dazu geschätzte $3 \sigma$ Konfidenzintervall für ein Einzelsystem. Deutlich erkennbar sind einzelne Spitzen beim Positionsfehler sowie ein fast durchgängig überschätztes Konfidenzintervall. 


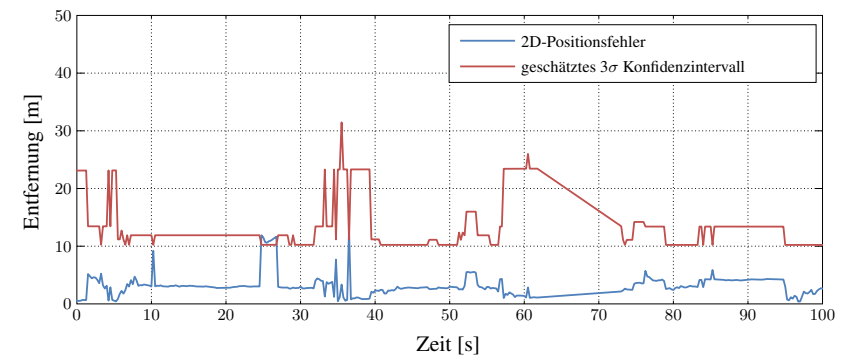

Abb. 6. Darstellung des 2-D-Positionsfehlers (blau) und des geschätzten $3 \sigma$ Konfidenzintervalls für ein Einzelsystem für einen Elevationswinkel von $30^{\circ}$.

Demgegenüber steht in Abbildung 7 ebenfalls der 2-DPositionsfehler und das $3 \sigma$ Konfidenzintervall, aber unter Verwendung beider Systeme zusammen. In diesem ist ein kleineres Konfidenzintervall zu sehen, womit sich der Algorithmus in seiner Lösung also sicherer wird. Zusätzlich werden einzelne Fehlerspitzen abgemildert. Bei Sekunde 58 zeigt sich ein extrem großer Ausreißer, der selbst mit beiden Systemen zusammen nicht abgemildert werden konnte.

\section{Zusammenfassung}

Die Positionierung mit Satellitensystemen bietet eine gute und kostengünstige Möglichkeit, sowohl in ruralen wie auch in urbanen Gebieten eine Position ermitteln zu können. In ruralen Gebieten ergeben sich durch die Zusammenführung von mindestens zwei Satellitensystemen zu einem Gesamtsystem nur wenig Verbesserungen, zeigen sie sich in einem urbanen Gebiet durch die Einschränkung des sichtbaren Bereiches zu den Satelliten dafür umso deutlicher. Es sind sowohl länger Positionslösungen möglich und es ergeben sich bei höheren Elevationswinkeln bessere Genauigkeiten. Zusätzlich kann die Standardabweichung herabgesenkt werden, wodurch der Lösung mit beiden Satellitensystemen zusammen mehr vertraut werden kann.

Mit fortschreitender Entwicklung weiterer Satellitensysteme, im Hinblick auf Galileo, wäre eine Untersuchung der weiteren Einflussnahme zusätzlicher Satellitensysteme auf die Positionslösung in urbanen Gebieten zu untersuchen.

\section{Literatur}

Artaud, G., de Latour, A., Dantepal, J., Ries, L., Senant, E., Maury, N., Denis, J.-C., and Bany, T.: A new GNSS multi constellation simulator: NAVYS, in: Satellite Navigation Technologies and European Workshop on GNSS Signals and Signal Processing (NAVITEC), 2010 5th ESA Workshop on, 1-8, 2010.

Cai, C.: A Solution for Combined GPS/GLONASS Navigation in Conditions of Limited Satellite Visibility, in: Proceedings of the 21st International Technical Meeting of the Satellite Division of

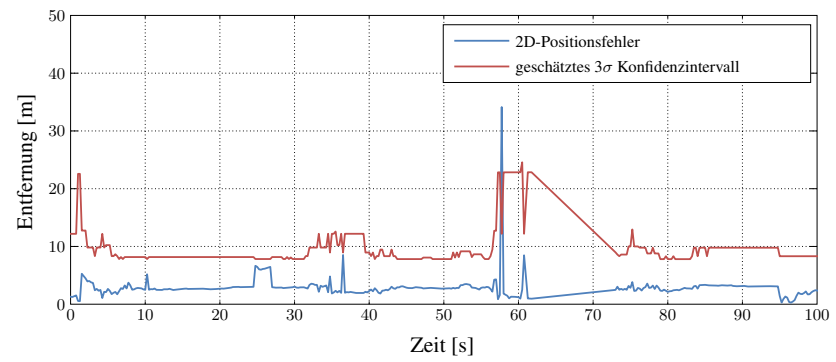

Abb. 7. Darstellung des 2-D-Positionsfehlers (blau) und des geschätzten $3 \sigma$ Konfidenzintervalls unter Verwendung beider Satellitensysteme als ein Gesamtsystem für einen Elevationswinkel von $30^{\circ}$.

The Institute of Navigation (ION GNSS 2008), 1398-1405, Savannah International Convention Center, Savannah, GA, 2008.

Delmas, P., Tessier, C., Debain, C., and Chapuis, R.: GNSS bias correction for localization systems, in: Proc. 11th Int Information Fusion Conf, 1-6, 2008.

Duchateau, G., Nouvel, O., Vigneau, W., Betaille, D., Peyret, F., and Secretan, H.: How to Assess and Improve Satellite Positioning Performances in Urban Environments, in: 16th ITS World Congress, Stockholm, 2009: Proceedings, 2009.

Engel, U.: Improving position accuracy by combined processing of Galileo and GPS satellite signals, in: Proc. 11th Int Information Fusion Conf, 1-8, 2008.

Juang, J.-C. and Tsai, Y.: On exact solutions of the multiconstellation GNSS navigation problem, GPS Solutions, 13, 5764, 2009.

Kaplan, E. D. and Hegarty, C.: Understanding GPS : Principles and Applications, Artech House, 2. Edn., 2006.

Mattos, P. G.: Accuracy and Availability Trials of the Consumer GPS/GLONASS Receiver in Highly Obstructed Environments, in: Proceedings of the 24th International Technical Meeting of The Satellite Division of the Institute of Navigation (ION GNSS 2011), 2740-2744, Oregon Convention Center, Portland, Oregon, 2011.

Miaoyan, Z., Jun, Z., and Yong, Q.: Satellite selection for multiconstellation, in: Proc. IEEE/ION Position, Location and Navigation Symp, 1053-1059, 2008.

Nahimana, D. F., Duflos, E., and Marais, J.: Reception state estimation of GNSS satellites in urban environment using particle filtering, in: Proc. 11th Int Information Fusion Conf, 1-5, 2008.

Rizos, C.: Multi-Constellation GNSS/RNSS from the perspective of high accuracy users in Australia, J. Spat. Sci., 53, 29-63, 2008.

Schubert, R., Richter, E., Mattern, N., Lindner, P., and Wanielik, G.: Advanced Microsystems for Automotive Applications 2010 - Smart Systems for Green Cars and Safe Mobility, chap. A Concept Vehicle for Rapid Prototyping of Advanced Driver Assistance Systems, 211-219, Springer, 2010.

Xu, L., Li, X., and Xue, Y.: Two Methods of Processing System Time Offset in Multi-constellation Integrated System, in: Electrical Engineering and Control, edited by Zhu, M., Vol. 98 of Lecture Notes in Electrical Engineering, 359-365, Springer Berlin Heidelberg, 2011. 